\title{
Neuer Test zur Beurteilung der Lebensqualität
}

Jones PW et al. Development and first validation of the COPD assessment test.

Eur Respir J. 2009;34:648-654

\section{Hintergrund}

Zulassungsbehörden und Einrichtungen, die medizinische Qualität bewerten, fordern zum Nachweis eines Therapieansatzes neben harten diagnostischen Ergebniskriterien auch die Erfassung von patientenbezogenen Parametern. Dies geschieht in der Regel mithilfe von vom Patienten auszufüllenden Fragebögen zur Lebensqualität oder zu den Symptomen. Die Fragebögen sind aber mitunter lang, kompliziert, zeitraubend auszufüllen, schwierig auszuwerten und eignen sich in praxi kaum.

\section{Fragestellung}

Nachdem der Asthmakontrolltest (ACT) mit seinen insgesamt fünf Fragen sehr anwenderfreundlich ist [1], wird mit dem COPD-Assessment-Test (CAT) nun ein entsprechender Test für die COPD entwickelt, dessen erste Ergebnisse jetzt vorgestellt wurden.

\section{Kriterien im COPD- Kontrolltest \\ 1 Husten \\ 2 Auswurf \\ 3 Enge auf der Brust \\ 4 Luftnot \\ 5 Reduktion der Alltagsaktivitäten \\ 6 Selbstvertrauen, das Haus bzw. die Wohnung verlassen zu können \\ 7 Schlafqualität \\ 8 Abgeschlagenheit}

Jede Frage wird abgestuft in einer Fünferskala bewertet. Insgesamt wären für alle acht Fragen o-40 Punkte möglich. Eine hohe Punktzahl bedeutet eine schlechte und eine niedrige Punktzahl eine gute Bewertung. Der Fragebogen ist kostenfrei online verfügbar unter: www.catestonline.org

\section{Ergebnisse}

Von ursprünglich 21 Fragen sind nach diversen Testdurchläufen noch acht Fragen im CAT übrig geblieben (Tab. 1). Als Goldstandard wurde der St. George's Respiratory Questionnaire (SGRQ) verwendet, gegenüber dem der CAT eine Korrelation von $r=0,8$ aufwies.

Der CAT kann zwischen stabiler und exazerbierter COPD gut unterscheiden ( $p<0,0001)$, wie eine Testreihe an 296 Patienten belegte (Abb. 1).

\section{Kommentar}

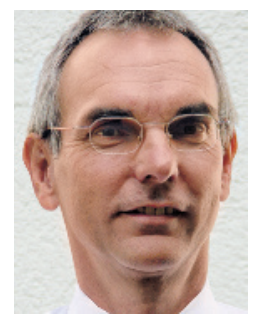

Prof. Dr. med. Adrian Gillissen, Leipzig
Die Entwicklung eines kurzen und verlässlichen Fragebogens zur Lebensqualität von COPD-Patienten ist wegen der Vielschichtigkeit der Erkrankung ungleich schwieriger als beim Asthma. Was der CAT in der Betreuung von COPD-Patienten zu leisten vermag, muss noch geklärt werden. Ungelöste Fragen sind z.B.:

- Ist mithilfe des CAT eine Therapiesteuerung möglich?

- Welchen Stellenwert hat der CAT im Konzert mit der übrigen uns zur Verfügung stehenden Diagnostik (Lungenfunktionsprüfung, Belastungstests)?

_Eignet er sich zur Prognoseabschätzung?

- Ist er zur inter- oder nur zur intraindividuellen Verlaufsbeurteilung nutzbar?

_Wo sind seine Grenzen?

\section{Fazit}

Der CAT ist nicht für die COPD-Diagnostik validiert. Er erlaubt allerdings eine wesentlich bessere Quantifizierbarkeit des Allgemeinbefindens des Patienten, als dies bisher möglich war, und hat damit einen gegenüber den übrigen diagnostischen Verfahren unterstützenden Wert.

\section{Literatur}

1. Schatz $M$ et al. Asthma control test: reliability, validity, and responsiveness in patients not previously followed by asthma specialists. J Allergy Clin Immunol. 2006;117:549-556

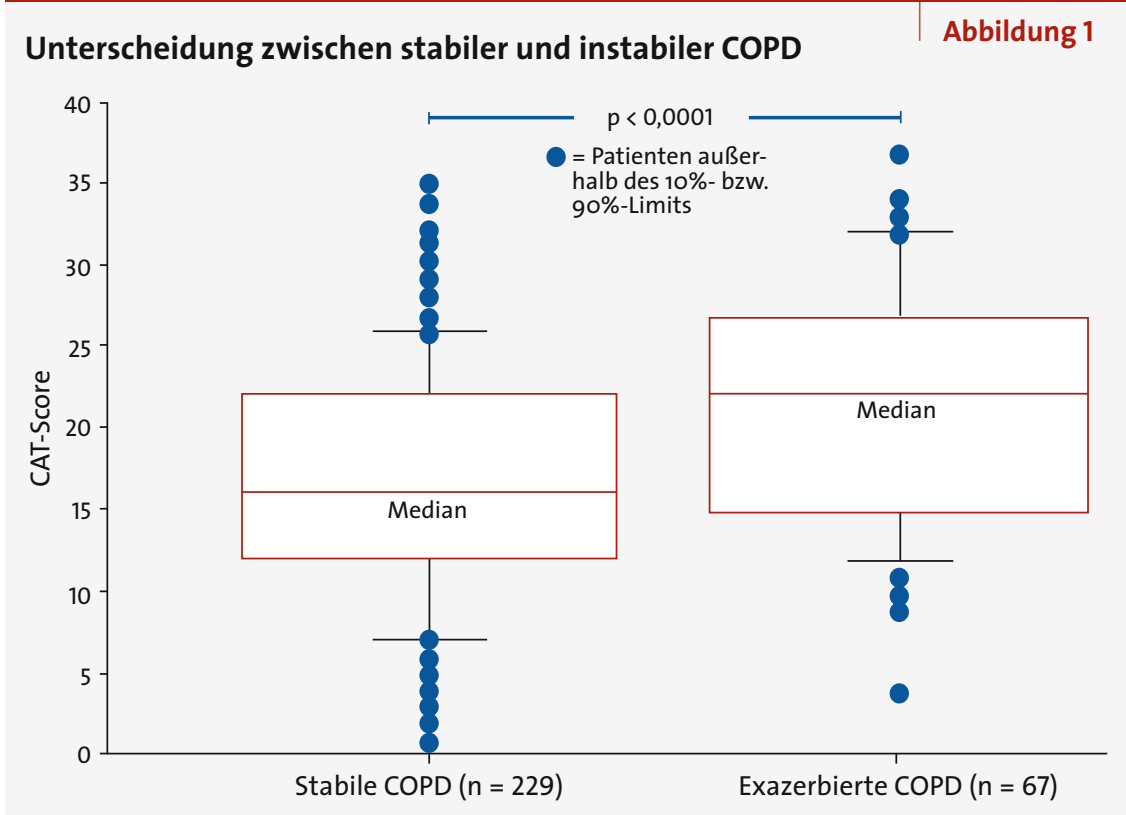

